\title{
Angiogenesis Induction using Dental Pulp Stem Cells, A Collagen Scaffold Seeded in Pulp Chamber of Endodontically Treated Teeth and Subcutaneously Transplanted into Immunocompromised Mice
}

Research Article

Yindeedej $\mathrm{W}^{1}$, Manotham $\mathrm{K}^{2 *}$, Chattong $\mathrm{S}^{2}$, Kiatsomchai $\mathrm{P}^{2}$, Ploypradith $\mathrm{D}^{1}$

${ }^{1}$ Dental Implant Unit, Department of Dentistry, Lerdsin General Hospital, Bangkok, Thailand.

${ }^{2}$ Renal Unit, Department of Medicine, Lerdsin General Hospital, Bangkok, Thailand.

\section{Abstract}

Background: Angiogenesis is an essential mechanism for repairing any damaged target tissues or organs in cell therapy because the process can provide the supplements of nutrients, oxygen, and other repair factors. Many studies have revealed the ability of stem cells to induce angiogenesis of vessels in various organs. According to previous studies, We postulated that dental pulp stem cells (DPSCs) may improve angiogenesis for dental tissue repair and hence the outcome of regeneration. Objective: We would like to evaluate the potential of DPSCs to induce an angiogenesis for dental tissue repair and regeneration.

Methods: In this study we employed endodontically treated teeth as a model to evaluate the angiogenesis properties of dental pulp stem cells. DPSCs were isolated from human third molar impacted tooth samples and reconstituted in basement membrane matrix scaffold (Matri-Gel 3D) and injected in the pulp chamber of the prepared endodontically treated teeth. Transplantation of experimental and control teeth was performed subcutaneously in the dorsum of immunocompromised mice (NOD-SCID).

Results: The vascular numbers in subcutaneous connective tissues of 4 week experimental mice, DPSCs and Matri-Gel scaffolds appeared visibly greater than those in the control group with Matri-Gel transplantation alone. Similar appearance was also found in the section of H-E stain of soft tissues of experimental teeth.

Conclusions: This may suggest that DPSCs can induce the regeneration of vascular dependent tissues such as dental pulp regeneration in necrotic pulp from diseases and may have implications in the regeneration of revital tooth.

Keywords: Dental Pulp Stem Cell; Angiogenesis; Immunocompromised Mice; Matrix Scaffold.

\section{Introduction}

A stem cell is commonly defined as a cell that is capable of self-renewal, continuous division and differentiation into other various types of cells or tissues. The dental stem cells from dental pulp of adult teeth, first found by Gronthos et al., [1] and called the dental pulp stem cells (DPSCs), can generally be found in pulp tissues of postnatal human permanent tooth. The DPSCs were described as mesenchymal stem cells (MSCs), with high potential proliferate and regenerate dental structures both in vivo and in vitro. The other dental stem cells from human exfoliated deciduous teeth (SHED) in young adult were found and also process high potential closely resembling that of MSCs, and as well as in Embryonic stem cells [2]. Currently, the periodontal ligaments of tooth represent a new source of stem cells [3]. Several studies investigated dental stem cells for their basic and advance biological properties and phenotype, and also in vivo and in vitro applications for cell therapy using dental tissues and other organs [1-5].

In stem cell therapy, an employment of proper stem cells, an essential scaffold and molecular factor as the three main parts [5]. However angiogenesis is also an essential mechanism for repair-

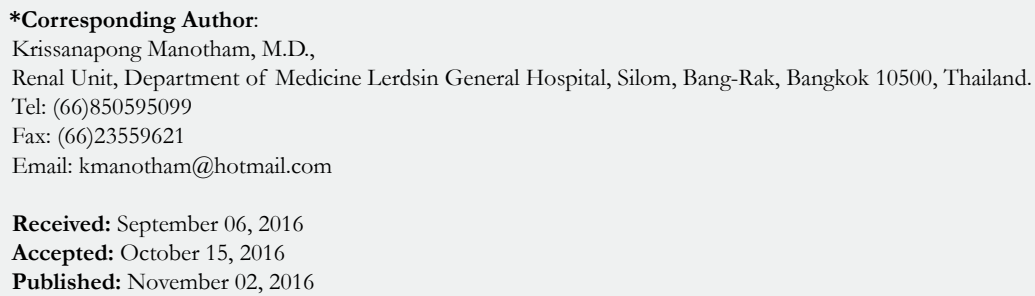

Citation: Yindeedej W, Manotham K, Chattong S, Kiatsomchai P, Ploypradith D (2016) Angiogenesis Induction using Dental Pulp Stem Cells, A Collagen Scaffold Seeded in Pulp Chamber of Endodontically Treated Teeth and Subcutaneously Transplanted into Immunocompromised Mice. Int J Stem Cell Res Transplant. 4(9), 251-255. doi: http://dx.doi.org/10.19070/2328-3548-1600038

Copyright: Manotham $\mathbf{K}^{\circ}$ 2016. This is an open-access article distributed under the terms of the Creative Commons Attribution License, which permits unrestricted use, distribution and reproduction in any medium, provided the original author and source are credited. 
ing damaged tissues [6]. The regeneration of damaged tissues can occur following angiogenesis, as the process provided nutrients, oxygen, and others repair factors. Many studies have revealed the ability of stem cells to induce angiogenesis of vessels in various organs such as heart vessels or in the experimental graft tissues [6, 8]. The improvement of revascularization of the new vessels may occur via the repaired mechanism of damage tissues or organs. The dental pulp stem cells could also secrete multiple angiogenic factors and could have therapeutic potential in the repair mechanism $[8,9]$.

The cell therapy employing the unipotential dental stem cells is to produce a new tooth or to repair damages of the tooth structures [5]. For dental pulp regeneration, many studies have been performed to create a practical endodontic tissue-engineering therapy for repairing or producing pulp tissues by using stem cell therapy. Investigators have devised dental pulp stem cells into different types of designed scaffolds, the three dimensional structure, as well as combined them with several growth factors or other chemicals. Prescott and colleague reported the use of dental pulp stem cells together with a collagen scaffold and dentin matrix protein1 (DMP1) which were incorporated into the prepared human tooth slices of $2.5 \mathrm{~cm}$. thickness [10]. After 6 weeks, they were transplanted into the subcutaneous dorsum of the immunocompromised mice, a new tissue resembling pulp tissues with new vessels was found. The same results were also found by using human exfoliated deciduous teeth (SHED) with biodegradable collagen scaffolds prepared within human tooth slices without any growth factors and transplanted into the subcutaneous dorsum of the immunocompromised mice [11]. The present resulting tissue closely resembled a dental pulp-like tissue. Both studies in vitro and in vivo of dental pulp stem cells have recently prompted many centers of dental research to revise further research with the aim to better understand and create new knowledge of how to use stem cells as a new way for dental treatments $[7,12,13]$.

The objective of this study is to measure the effectiveness of DPSCs combined with basement membrane matrix scaffold (MatriGel 3D) without growth factor, in the in-vivo generation of the induced new vessels or angiogenesis following subcutaneous transplantation of endodontically treated tooth as a model study in the immunocompromised mice.

\section{Materials and Methods}

\section{DPSCs Isolation and Culture}

Normal human impacted third molars were collected from adults (19-25 years of age) at the Dental Department of Lerdsin Hospital under approved guidelines set by the Lerdsin Ethics committee and Lerdsin Research committee. Tooth surfaces were cleaned and cut around the cemento-enamel junction by using sterilized dental fissure burs to reveal the pulp chamber. The pulp tissue was gently separated from the crown and roots. The procedure of stem cell isolation was modified as previously described [1]. Breifly, the pulp tissue was removed from the pulp chamber under steriled technique and enzymatically digested by a solution of 3 $\mathrm{mg} / \mathrm{ml}$ collagenase type I (Gibco, Grand Island, NY, U.S.A.) for $1 \mathrm{~h}$ at $37^{\circ} \mathrm{C}$. The digestion was stopped by adding $5 \mathrm{ml}$ of $10 \%$ FBS DMEM supplemented with 2 mM L-glutamine, 100 units/ $\mathrm{mL}$ penicillin $\mathrm{G}$-streptomycin and centrifugation at $1200 \mathrm{rpm}$ for
$10 \mathrm{~min}$ at $4^{\circ} \mathrm{C}$. The cell pellet was resuspended in above culture medium and then plated into $10 \mathrm{~cm}$ petridish. The cell culture was incubated in air with $5 \% \mathrm{CO}_{2}$ at $37^{\circ} \mathrm{C}$. The culture medium was changed every three days until reaching cell confluence.

\section{Flow Cytometry for Surface Marker Characterization of DPSCs}

Cells were trypsinized and resuspended in PBS at density of $1 \mathrm{X}$ 107 cells $/ \mathrm{mL}$, and then $100 \mu \mathrm{l}$ of cell suspension was incubated with FITC-, or PE-, PE-Cy 7 conjugated monoclonal antibodies against CD 34, CD 45, HLA-DR, HLA-ABC, CD79a, CD 29, CD 33, CD 44, CD 73, CD 90 and CD 11b (BD Biosciences, Bedford, MA). Isotype-match PE- or FITC- or PE-Cy7 conjugated mouse antibodies were used as controls. These cells were incubated for 30 minutes in dark room at room temperature. After incubation, excess antibodies were washed off by adding PBS and centrifugation at $2000 \mathrm{rpm}$ for $5 \mathrm{~min}$. Antibody incubated-cells were resuspended in $200 \mu \mathrm{l} \mathrm{FAC} \mathrm{solution} \mathrm{and} \mathrm{flow} \mathrm{cytometry} \mathrm{was}$ performed by using a FACS Calibur (BD Biosciences, San Diego, USA). The data were analyzed with Cell Quest Pro software (BD Biosciences).

\section{Reverse Transcription-PCR}

Total RNA was extracted from DPSCs at passage 2 with Purezol (Biorad, Hercules, CA, U.S.A.) according to the manufacturer's protocol. cDNA synthesis was performed by using the Protoscript@ first strand cDNA synthesis kit (New England Biolabs, Ipswich, MA, U.S.A.). One microgram of cDNA was used for PCR with the corresponding primers Oct4- sense GGA AGG TAT TCA GCC AAA CG antisense CCC TGA GAA AGG AGA CCC A, SOX2- sense CCA GCG CAT GGA CAG TTA C antisense TGG AGT GGG AGG AAG AGG TA, ERAS- sense CAA CAA AGC CTG GCA CCT antisense CAG CAG TGG TCA CAA GGT CA, REX1- sense AAC GGG CAA AGA CAA GAC AC antisense TCC AAA GAA CAT TCA AGG GAG, KLF4- sense GCT TCA CCT ATC CGA TCC antisense CAG CAC GAA CTT GCC CAT, NANONG- sense ACC AGT CCC AAA GGC AAA C antisense GAG TAA AGG CTG GGG TAG GTA G with Stratagene Mx 3000p (Stratagene, La Jolla, CA, U.S.A.) and EXTaq DNA polymerase (Takara, Shiga, Japan). The PCR products were electrophoresed and visualized under UV light.

\section{Tooth Model Structure Preparation}

Ten human extracted wisdom teeth from surgical removal of the impacted third molar teeth after receiving an approval on the consent forms of the Dental Department of Lerdsin Hospital. The teeth were examined under an operating microscope (3X Heines Dental Loop) to detect possible defects such as root or crown fractures. Radiographs of each tooth were assessed to ensure that each had a single root canal. Using a high speed diamond bur with normal saline coolant, the root was cut approximately at onethird of the root length. Subsequently, the root canal was drilled with a small round diamond bur to provide an access to the pulp chamber. The pulp chamber was cleaned and shaped according to the root canal of teeth using rotary instruments. The instruments of shaping the root canals with increasing size employed the following sequence of files: No.14-40 K-File NITIFLEX Dentsply, 
USA. During cleaning and shaping, each tooth was irrigated with a $6 \%$ sodium hypochlorite $(\mathrm{NaClO})$ solution (Clorox) and $10 \%$ hydrogen peroxide following the use of each instrument size. In the last step, the procedure was followed by a one- minute application of $3 \mathrm{~mL}$. of $17 \%$ ethylenediaminetetraacetic acid (EDTA) and a final flush with $6 \mathrm{~mL}$ of $6 \% \mathrm{NaClO}$. After cleaning and shaping the root canal and pulp chamber of the teeth, the tooth was disinfected by $6 \% \mathrm{NaClO}$ for five minutes, and autoclaved at $134^{\circ} \mathrm{C}$ for 20 minutes. The specimens were then washed in the sterile PBS solution before cell seeding in following step.

\section{Cell Seeding and Transplantation of the Experimental} Tooth into the Immunodeficiency Mice

1 X $10^{5}$ cells were resuspended in a 1:1 mixture of Matri-Gel (BD Biosciences, Bedford, MA) and cell culture medium. The mixture of cells was seeded into the tooth structure via apical root canal, the tooth-containing cells were incubated for 30 minutes at $37^{\circ} \mathrm{C}$ to allow for the settling of the Matri-Gel. Control tooth structure was filled with 1:1 mixture of Matri-Gel and cell culture medium without cells.

Immunodeficiency mice (NOD-SCID), 5-7 week ( $\mathrm{n}=10)$ were weighed and anesthetized with ether gas inhalation in an induction chamber, with anesthesia maintained with a nose cone ether. A $2 \mathrm{~cm}$-long linear incision was made along the lateral side of the dorsum of mice. Teeth were implanted in the subcutaneous pocket superior to dorsal muscles, on the right side for the experimental tooth and left for the control. All teeth were harvested after 4 weeks by removing external skin, subcutaneous connective tissues and the surrounding fibrous capsule, and teeth were fixed in $10 \%$ buffered formalin for 24 hours, demineralized with $10 \%$ nitric acid at room temperature for 7-10 days until the dentin of the teeth offered no resistance to cutting. The demineralized teeth were processed and embedded in paraffin blocks. The blocks were sectioned in the transverse plane at $5 \mathrm{~mm}$. thickness and stained with hematoxylin and eosin (H\&E).

\section{Results}

\section{DPSCs Expressed Fibroblast - Like Morphology and Pro- tein Markers of Mesenchymal Stem Cell}

When the pulp tissues were separated from the crown and roots (Figure 1a). After isolation and culture, DPSCs became the clonogenic form within 3 days (Figure 1b) and these cells were confluent within 10 days (Figure 1c).

Surface markers of DPSCs were characterized by flow cytometric analysis. As shown in Figure 1C, DPSCs showed a mesenchymal stem cell (MSCs) expression pattern upon their interactions with a large panel of antibodies. DPSCs showed positive expression to CD 29, CD 33, CD 44, CD 73, CD 90 and CD HLA-ABC while negative to CD 33, CD 34, CD 45, CD 79a and HLA-DR. This result suggested that DPSCs showed the characteristic of MSCs (Figure 1d).

\section{Reverse Transcription-PCR also Showed that DPSCs Ex- pressed Important Markers of Stem Cells}

DPSCs were found to express NANOG and SOX2, two impor- tant markers of stem cells. These results suggested that DPSCs are similar to other mesenchymal stem cells. (Figure 2).

\section{In vivo study of DPSCs in NOD-SCID}

In vivo vascularization was observed in DPSCs combined with Matri-Gel scaffolds: When the experimental teeth that generated DPSCs and Matri-Gel scaffolds were implanted subcutaneously into the dorsum of nude mice and were evaluated for tissue generation. Four weeks after implantation, the DPSCs combined with Matri-Gel tooth demonstrated notably higher visible vascularization surrounding the connective tissues and in the vicinity of the apical root of the experimental tooth (Figure 3a). In comparison, only limited vascularization was observed surrounding control tooth with Matri-Gel alone without DPSCs. (Figure 3b).

\section{DPSCs Combined with Matri-Gel Scaffolds Induced a For- mation of Connective Tissue and Red Blood Cells}

In $\mathrm{H} \& \mathrm{E}$ section we found loose connective tissues consisting of DPSCs and endothelial-like structure were found in some areas of the experimental specimens. In addition, in the lumen-like structure were found some red blood cells. (Figure $4 \mathrm{a}$ and $4 \mathrm{~b}$ ). In the control groups, we found only gel structure with no cellular structures (Figure 4c).

\section{Discussion}

In our study, we cannot find a pulp-like tissue in the pulp chamber of the experimental tooth transplanted in the immunocompromized mice, but new vessels at the apical area were visible in the experimental tooth which was given the DPSCs. None of the vessels were observed in the control tooth which was given only the scaffold alone without stem cells. In addition, loose connective tissues consisting of DPSCs and endothelial-like structure in $H \& E$ section were observed in some areas of the experimental specimens. In the lumen-like structure, we found some red blood cells. This suggested that DPSCs alone had the ability to induce new vessels formation or angiogenesis in the target organs.

We employed the prepared tooth structure as the model study instead of the tooth slices because the prepared tooth structure resembled were closely a realistic situation of cell therapy for pulp regeneration in pulp chamber and root canal. Following the classical endodontic treatment protocols and with addition of dental pulp stem cells efficiency of dental pulp stem cells in tissue regeneration was evaluated for a new optional treatment for revitalizing pulp. No growth factors nor any matrix proteins were utilized because these substances may influence to our cells and interfere with the experimental mechanisms. The growth factors might induce our cells instead while overwhelming or obscuring the influences of stem cells on the pattern of vascularization. New vessels were found to infiltrate at the apical of the experimental tooth. New vessels formations indicated that repair mechanism can occur. Although the exact mechanism has not been fully characterized, some studies found that MSCs could induce angiogenesis by excreted or induced angiogenic cytokines such as vascular endothelial growth factor (VEGF), platelet-derived growth factor (PDGF) and fibroblast growth factor (FGF) [6-8]. DPSCs expressed MSCs-like properties, so it may induced angiogenesis in 
Figure 1. Morphology and Surface Marker Expression of DPSCs.
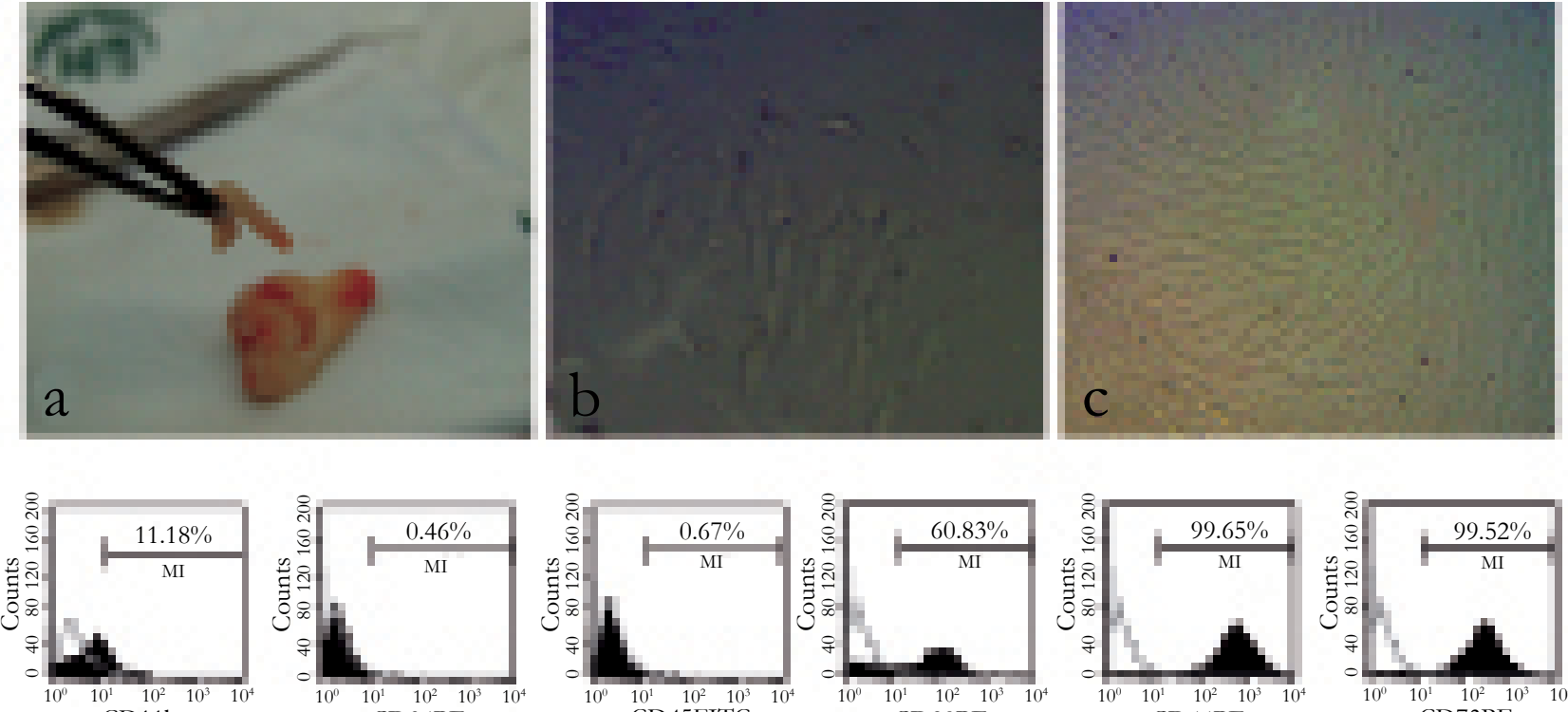

$\mathrm{CD} 11 \mathrm{~b}$

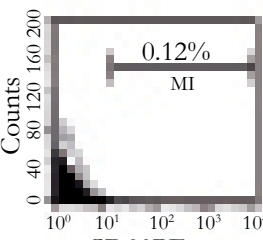

CD33PE

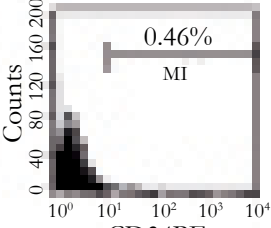

CD34PE

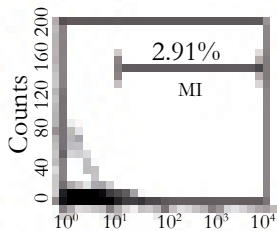

$\mathrm{CD} 79 \mathrm{aPE}$

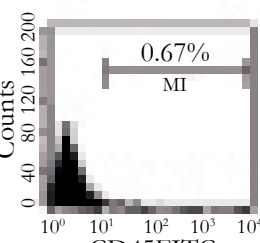

CD45FITC

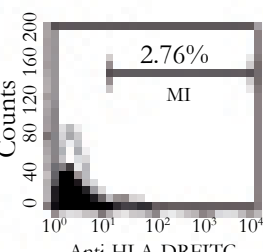

Anti-HLA-DRFITC

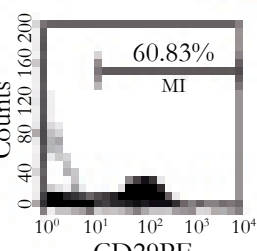

CD29PE

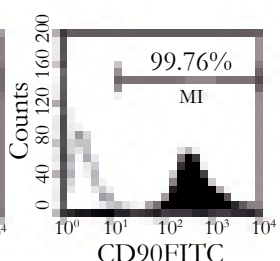

CD90FITC

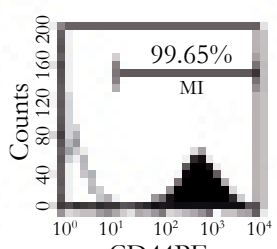

CD44PE

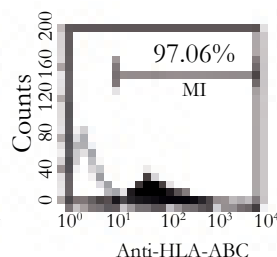

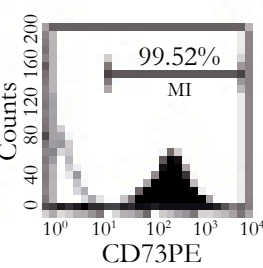

CD73PE

After isolation pulp tissues (a) and culture, DPSCs became clonogenic form at day 7 (b) and confluent within 10 days (c). DPSCs showed a mesenchymal stem cell (MSCs) expression pattern which positive to CD 29, CD 33, CD 44, CD 73, CD 90 and CD HLA-ABC while negative to CD 34, CD 45, CD 79a and HLA-DR (c).

Figure 2. DPSCs Expressed Important Markers of Stem Cells.

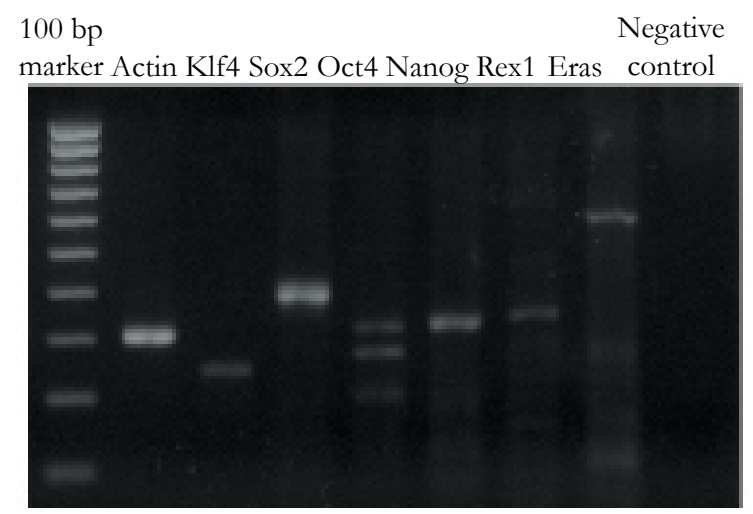

DPSCs were found to express NANOG and SOX2, two important markers of stem cells. These results suggested that DPSCs are similar to other mesenchymal stem cells.

Figure 3. DPSCs Combined with Matri-Gel scaffolds Induced In vivo Vascularization.

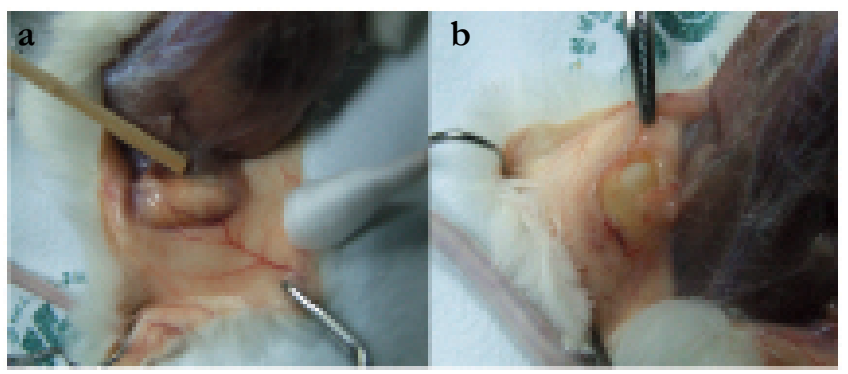

After implantation for 4 weeks, the DPSCs combined with Matri-Gel tooth demonstrated notably higher visible vascularization surrounding the connective tissues and in the vicinity of the apical root of the experimental tooth (a). In comparison, only limited vascularization was observed surrounding control tooth with Matri-Gel alone without DPSCs. (b). 
Figure 4. DPSCs Combined with Matri-Gel Scaffolds Induced a Formation of Connective Tissues and Red Blood Cells.

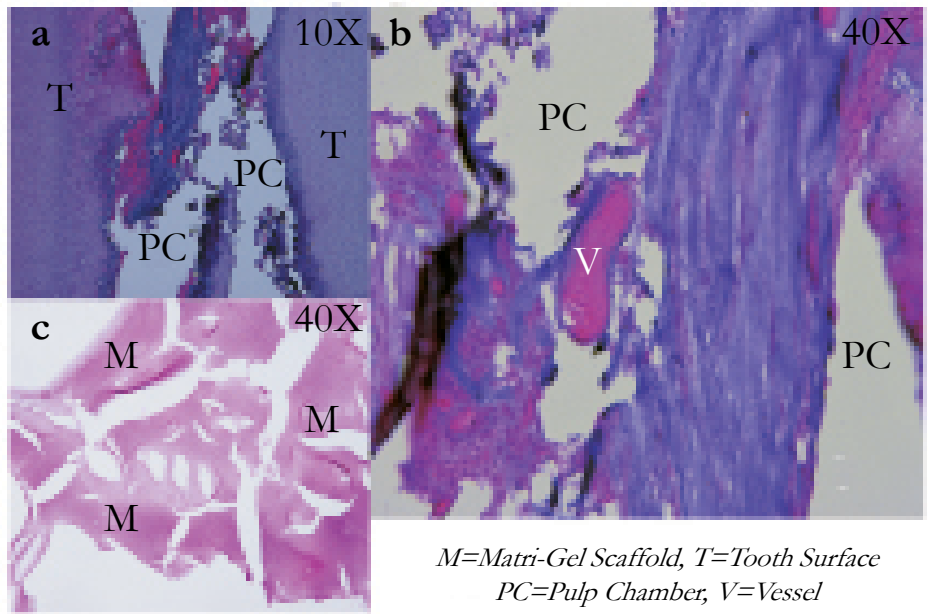

In H\&E section, the loose connective tissues consisting of DPSCs and endothelial-like structure were found in some areas of the experimental specimens. In addition, in the lumen-like structure were found some red blood cells. (a and b). In the control groups, we found only gel structure with no cellular structures (c).

the target organ by means of induced endothelial and associated growth factors [14-18]. The results of our study are in good accordance with this hypothesis. Gandia and colleagues showed that DPSCs improved left ventricular function by inducing angiogenesis and could reduce infarct size in rats with acute myocardial infarction, using DPSCs alone without the use of any other factors [9].

In our study, we used DPSCs alone without any other factors to evaluate the angiogenic property of stem cells, while avoiding the effects of those factors that may influence or obscure our experiments. A clear advantage of increasing new vessels appears to indicate that tissue repair can occur and tissue regeneration is plansible. Further studies are warranted for further success in dental pulp regeneration using stem cell therapy.

\section{References}

[1]. Gronthos S, Mankani M, Brahim J, Robey PG, Shi S (2000) Postnatal human dental pulp stem cells (DPSCs) in vitro and in vivo. Proc Natl Acad Sci USA. 97(25): 13625-13630.

[2]. Miura M, Gronthos S, Zhao M, Lu B, Fisher LW, et al., (2003) SHED: Stem cells from human exfoliated deciduous teeth. Proc Natl Acad Sci USA. 100(10): 5807-5812.

[3]. Seo BM, Miura M, Gronthos S, Bartold PM, Batouli S, et al., (2004) Investigation of multipotent postnatal stem cells from human periodontal ligament. Lancet. 364(9429): 149-155.

[4]. Suchanek J, Soukup T, Visek B, Ivancakova R, Kucerova L, et al., (2009) Dental pulp stem cells and their characterization. Biomed Pap Med Fac Univ Palacky Olomouc Czech Repub. 153(1): 31-36.

[5]. Bluteau G, Luder HU, De Bari C, Mitsiadis TA (2006) Stem cells for tooth engineering. Eur Cell Mater. 16: 1-9.

[6]. Moioli KE, Clark AP, Mo C, Dennis EJ, Erickson P, et al., (2008) Synergistic actions of hematopoietic and mesenchymal stem/progenitor cells in vascularizing bioengineeried tissues. PLoS ONE. 12: e3922.

[7]. Laino G, Carinci F, Graziano A, d'Aquino R, Lanza V, et al., (2006) In vitro bone production using stem cells derived from human dental pulp. J Cranifac Surg. 17(3): 511-515.

[8]. Fang D, Seo MB, Yi L, Sonoyama W, Yamaza T, et al., (2007) Transplantation of Mesenchymal stem cells is an optimal approach for plastic surgery. Stem Cell. 25(4): 1021-1028.

[9]. Gandia C, Arminan A, Garcia-Verdugo MJ, Lledo E, Ruiz A, et al., (2008) Human dental pulp stem cells improve left ventricular function, induce angiogenesis, and reduce infarct size in rats with acute myocardial infarction. Stem cells. 26(3): 638-645.

[10]. Rebecca S Prescott RA, Mohamed IF, Bradford RJ, Christopher SW, John AS, et al., (2008) In-vivo generation of dental pulp-like tissue using human pulpal stem cells, a collagen scaffold and dentin matrix protein 1 after subcutaneous transplantation in mice. J Endod. 34(4): 421-426.

[11]. Cordiero MM, Dong Z, Kaneko T, Zhang Z, Miyazawa M, et al., (2008) Dental pulp tissue engineering with stem cells from exfoliated deciduous teeth. J Endod. 34(8): 962-969.

[12]. Eric LG, Peter EM, Kenneth NN, Sergio K, Franklin GG (2008) An ultrastructural investigation of tissue-engineered pulp constructs implanted within endodontically treated teeth. J Am Dent Assoc. 139(4): 457-465.

[13]. Sally L, Arnaud M, Patricio D, Dominique S, Laurent B, et al., (2008) An in vivo model for short-term evaluation of the implantation effects of biomolecules or stem cells in the dental pulp. Open Dent J. 2: 67-72.

[14]. Tran-Hung L, Mathieu S, About I (2006) Role of human pulp fibroblasts in angiogenesis. J Dent Res. 85(9): 819-823.

[15]. Matsushita K, Motani R, Sakuta T, Yamaguchi N, Koga T, et al., (2000) The role of vascular endothelial growth factor in human dental pulp cells: induction of chemotaxis, proliferation and differentiation and activation of the AP-1-dependent signaling pathway. J Dent Res. 79(8): 1596-1603.

[16]. Artese L, Rubini C, Ferrero G, Fioroni M, Santinelli A, et al., (2002) Vascular endothelial growth factor (VEGF) expression in healthy and inflamed human dental pulps. J Endod. 28(1): 20-23.

[17]. Piattelli A, Rubini C, Fioroni M, Tripodi D, Strocchi R (2004) Transforming growth factor beta 1 (TGF-beta1) expression in normal healthy pulps and in those with irreversible pulpitis. Int Endod J. 37(2): 114-119.

[18]. Mullane EM, Dong Z, Sedgley CM, Hu JC, Botero TM, et al., (2008) Effects of VEGF and FGF2 on the revascularization of severed human dental pulps. J Dent Res. 87(12): 1144-1148. 